\section{RMD Open}

Rheumatic \&

Musculoskeletal Diseases

\title{
IL-6-PAD4 axis in the earliest phase of arthritis in knock-in gp130F759 mice, a model for rheumatoid arthritis
}

Ayano Yahagi, ${ }^{1}$ Taro Saika, ${ }^{2}$ Hiroyasu Hirano, ${ }^{3}$ Miwa Takai-Imamura, ${ }^{4}$ Fumio Tsuji, ${ }^{5}$ Hiroyuki Aono, ${ }^{4}$ Masanori Iseki, ${ }^{1}$ Yoshitaka Morita, ${ }^{3}$ Hideya Igarashi, ${ }^{1}$ Yukihiko Saeki, ${ }^{6}$ Katsuhiko Ishihara ${ }^{1}$

To cite: Yahagi A, Saika T, Hirano $\mathrm{H}$, et al. IL-6-PAD4 axis in the earliest phase of arthritis in knock-in gp130F759 mice, a model for rheumatoid arthritis. RMD Open 2019;5:e000853. doi:10.1136/ rmdopen-2018-000853

- Additional material is published online only. To view please visit the journal online (http://dx.doi.org/10.1136/ rmdopen-2018-000853).

Received 8 November 2018 Revised 29 April 2019 Accepted 23 May 2019

\section{Check for updates}

(c) Author(s) (or their employer(s)) 2019. Re-use permitted under CC BY-NC. No commercial re-use. See rights and permissions. Published by BMJ.

For numbered affiliations see end of article.

Correspondence to Dr Katsuhiko Ishihara; ishihara-im@med.kawasaki-m. ac.jp

\section{ABSTRACT}

Objective Animal models for human diseases are especially valuable for clarifying molecular mechanisms before or around the onset. As a model for rheumatoid arthritis (RA), we utilise knock-in mice gp130F759. They have a Y759F mutation in gp130, a common receptor subunit for interleukin 6 (IL-6) family cytokines. Definitive arthritis develops around 8 months old and the incidence reaches $100 \%$ around 1 year old. Careful examination in the clinical course revealed very subtle resistance in flexibility of joints at 5 months old. Therefore, pathophysiological changes in gp130F759 were examined to dissect molecular mechanisms for preclinical phase of RA.

Methods Severity of arthritis in gp130F759 was evaluated with a clinical score system and histological quantification. Serum cytokines, autoantibodies and $\mathrm{C}$ reactive protein (CRP) were measured. Changes in the synovium were analysed by real-time PCR, flow cytometry and immunohistochemistry.

Results Around 5 months old, various types of cytokines, rheumatoid factor (RF), anti-circular citrullinated peptide IgM and CRP increased in the sera of gp130F759.

Enhancement of neovascularisation, synovial hyperplasia and fibrosis was observed. Also, increases in haematopoietic cells dominated by innate immune cells and gene expression of $/ / 6$ and Padi4 were detected in the joints. I/6 was expressed by non-haematopoietic synovial cells, whereas PAD4 protein was detected in the synovial neutrophils. Padi4 is induced in neutrophils in vitro by IL-6. Increases of phospho-STAT3 and PAD4 protein were detected in the synovium. Deletion of IL-6 in gp130F759 normalised the amount of PAD4 protein in the joints.

Conclusion The IL-6-PAD4 axis operates in the earliest phase of arthritis in gp130F759, implicating it in early RA.

\section{INTRODUCTION}

Rheumatoid arthritis (RA) is an autoimmune disease with $0.3 \%-1.5 \%$ morbidity. It is characterised by chronic, proliferative synovitis with bone erosion leading to joint destruction. Genetic factors (HLA-DRB1, PTPN22 and PADI4 genes, etc), as well as environmental factors (smoking and infection) are

\section{Key messages}

What is already known about this subject?

- Mutant gp130 knock-in mice gp130F759 are a model for rheumatoid arthritis (RA) where a point mutation of gp130 causes aberrant STAT3 activation.

What does this study add?

- The earliest time point was identified when the pathological changes common to human RA start in joints of gp130F759 mice.

- IL-6 dependent PAD4 production 'the IL-6-PAD4 axis', occurs in synovial neutrophils in the preclinical phase of arthritis in gp130F759 mice.

How might this impact on clinical practice?

- The gp130F759 model can now be used to probe very early stages of arthritis, seek novel therapeutic targets and devise new treatment strategies.

involved in breakdown of self-tolerance and activation of autoimmunity.

Critical findings in the understanding and treatment of RA are anti-citrullinated peptide autoantibodies (ACPAs) as specific for RA and effectiveness of anti-cytokine therapies. Development of biologics against TNF $\alpha$ or IL-6 has drastically changed the prognosis of RA and therapeutic strategies. Since serum ACPAs are detected much earlier than the onset of $\mathrm{RA}^{1-3}$ anti-circular citrullinated peptide (CCP) antibody was included in the criteria for RA of The European League against Rheumatism/American College of Rheumatology (EULAR/ACR) 2010. ${ }^{4}$ This permit earlier therapeutic application of biologics and the possibility of complete cures for this disease. Recent investigation of RA has proposed a stepwise, progressive model of RA. ${ }^{5-8}$ But it is difficult to obtain information on the molecular events in the synovium during preclinical phases of RA. This limitation can be partly overcome by the study of animal models. 
Citrullination of arginine is catalysed by peptidyl arginine deiminases (PADs), ${ }^{9}$ which results in generation of modified self-antigens and production of ACPA. PADI4 is one of the risk factors of RA identified by Genome Wide Association Study (GWAS). ${ }^{10}$ Single nucleotide polymorphisms (SNPs) of PADI4 is responsible for increased expression of PADI4, and deletion of Padi4 in mice ameliorated collagen-induced or anti-G6PI-induced arthritis. ${ }^{112}$ PAD4 mediates autoantibody production and inflammatory arthritis downstream of TNF $\alpha .{ }^{13}$ However, molecular mechanisms for expression of PADI4 before the clinical onset of RA are poorly understood.

IL-6 is a multifunctional cytokine that regulates the development and functions of neuronal, haematological, inflammatory and immunological systems. Dysregulation of IL-6 is involved in various autoimmune diseases including RA. ${ }^{14}$ IL-6 receptor complex consists of an IL-6 receptor $\alpha$ chain and a signal transducing subunit gp130. Gp130 is a common receptor subunit for the IL-6 family cytokines, transducing signals through two independent pathways, YXXQ/STAT3 and Y759/SHP2 pathways. ${ }^{15}$ Gp130F759 knock-in mice have a mutation of tyrosine to phenylalanine at the 759th amino acid residue, and a defect in the negative regulatory pathway by SOCS3. In turn, this leads to prolonged activation of STAT3 by physiological ligands. ${ }^{16}$ Aberrant activation of STAT3 results in splenomegaly, lymphadenopathy, hyper- $\gamma$ globulinaemia, auto-antibody production and RA-like arthritis with age. ${ }^{17}$ Gp130F759 is unique as providing evidence that one amino acid mutation in a cytokine receptor can cause an autoimmune disease. The development of arthritis in gp130F759 is Rag2-dependent, but the Y759F mutation in non-haematopoietic cells is sufficient. ${ }^{17}$ Several pathophysiological mechanisms have been reported. Homeostatic proliferation of $\mathrm{CD} 4^{+} \mathrm{T}$ cells driven by IL-6/STAT3-dependent IL-7 production by non-haematopoietic cells, ${ }^{18}$ IL-6-dependent acceleration of arthritis by transgene of HTLV- $1 p X^{19}$, triggering effects of polyclonal, antigen-non-specific Th17 accumulated in the joints, ${ }^{20}$ and a positive feedback loop of IL-6/IL-17A in chronic inflammation. ${ }^{21}$ From these studies, a fourstep model was proposed that explains the mechanism for tissue specific autoimmune diseases by antigen-non-specific activation of the IL-6 amplifier. ${ }^{22}$ This intriguing model is based on the experiments utilising artificial manipulations, such as neonatal thymectomy, ${ }^{18}$ puncture or injection of chemokine to the joints ${ }^{20}$ and over-expression of cytokines by plasmid injection. ${ }^{21}$ Arthritis of gp130F759 is characterised by late onset around 8 months old, equivalent to the predilection age of human RA, and gradually progresses. Therefore, we have exploited this model to learn that the IL-6-PAD4 axis is operative among early RA related changes.

\section{MATERIALS AND METHODS \\ Mice}

All mice in C57BL/ 6 background were maintained under specific pathogen free (SPF) condition in the animal facility in Kawasaki Medical School Central Research Institute. Gp130F759 is used as a notation for homotypic knock-in mice $g p 130^{F 759 / F 7591617}$ in this paper. Gp130F759 was crossed with $I l 6$ knockout mouse $(I l 6 \mathrm{KO})^{23}$ to make double mutant $I l 6 \mathrm{KO} / \mathrm{gp} 130 \mathrm{~F} 759$. The animal protocol for this experiment was approved by the Animal Care and Use Committee of Kawasaki Medical School.

\section{Scoring of arthritis}

The score system for arthritis from 0 to 4 points/a limb is based on the degree of restriction of joint flexibility $(0$, no restriction; 1 , slight; 2 , moderate; 3 , severe), and +1 point for redness or swelling for a joint of each fore-limb and hind-limb (online supplementary figure 1). Two researchers who are not informed about genotype scored independently.

\section{Cytokine measurement}

Concentration of serum cytokines from gp130F759 $(\mathrm{n}=12)$ and wild type (WT) $(\mathrm{n}=8)$ were measured using Bio-Plex (Bio-rad, Hercules, California, USA). IL-6 and $\mathrm{TNF} \alpha$ in culture supernatant of fibroblast-like synoviocytes were measured with ELISA kits for these cytokines (BioLegend, San Diego, California, USA) following manufacturers instruction.

\section{Neutralising antibody for IL-6}

Rat anti-IL-6 monoclonal antibodies (mAb) (IgG1; clone \#MP5-20F3) and isotype matched control mAb were purchased from BioLegend and Zymed (San Francisco, California, USA), respectively.

\section{Quantitative histology for arthritis}

Ankle containing synovium was fixed in $10 \%$ neutral buffered formalin, decalcified with $10 \%$ formic acid and embedded in paraffin. Sliced tissue was stained with HE. For estimation of fibrosis, sliced tissue was stained with Sirius red F3BA (WALDECK, Münster, Germany). Using Image $\mathrm{J}$ software available on the web site, histological changes in preclinical to very early arthritis were quantitatively estimated using criteria as follows (figure 2B): For neovascularisation, the numbers of the transverse section typical for newly formed vasculature (black arrow) in the total region of synovium (light green lined) were counted. Hyperplasia was defined by synovial lining that has four cell-layers or more, and evaluated with a formula (the length of lining with hyperplasia (yellow line)/length of total synovial lining (blue line) $) \times 100(\%)$. For fibrosis, the ratio of fibrosis (fibrosis area positively stained with Sirius $\mathrm{red} /$ total area of synovium (light green lined) $) \times 100(\%)$ was calculated by Image $J$ software.

\section{Cell separation}

Splenocytes were prepared by teasing the spleen with slide glasses. The synovium of the knee joint was dissected with scalpel, minced and digested with collagenase D (Roche Diagnostics, Mannheim, Germany) for 3 hours at $37^{\circ} \mathrm{C}$ in $\mathrm{CO}_{2}$ incubator. After washing and passing through mesh, synovial cells were used for flowcytometry.

Synovial cells were separated to $\mathrm{CD} 45^{+}$haematopoietic and $\mathrm{CD} 45^{-}$non-haematopoietic cells using rat 
anti-mouse CD45 mAb (BD Biosciences) and magnetic beads coupled with sheep anti-rat IgG $(\mathrm{H}+\mathrm{L})$ (Thermo Fisher Scientific,Waltham, MA). Real-time PCR detected no transcripts for $C d 45$ in the cDNA prepared from $\mathrm{CD} 45^{-}$fractions. Cultured for 3 days in MEM $\alpha$ containing $15 \%$ Fetal calf serum (FCS) and penicillin/streptomycin, CD $45^{-}$fractions showed typical morphology for fibroblasts, indicating that synovial $\mathrm{CD} 45^{-}$fractions are compatible with fibroblast-like synoviocytes.

Bone marrow neutrophils were purified with neutrophil separation kit (Miltenyi Biotech, Bergisch Gladbach, Germany) using MACS LS column. Purities of neutrophils $\left(\mathrm{CD} 11 \mathrm{~b}^{+} \mathrm{Gr}-1^{\text {hi }}\right)$ confirmed with FACSCanto II were usually more than $98 \%$. Neutrophils were stimulated with various concentrations of human rIL-6 (2-100 ng/mL), LPS (E. coli 0111:B4) (Sigma-Aldrich, St. Louis, Missouri, USA), or culture supernatant fluid of $\mathrm{CD} 45^{-}$synovial cells for 6 hours. After each separation or incubation, the cells were collected into TRIzol (Thermo Fisher Scientific) and store at $-80^{\circ} \mathrm{C}$ until use.

\section{Flow cytometry and typing of synovial cells}

Splenocytes and synoviocytes were blocked with mixture of supernatant of anti-CD16/32 mAb (2.4G2) and $5 \%$ heat inactivated rat serum. Then the cells were stained with two sets of cocktail mAbs labelled with fluorescent dyes. Set 1 consists of FITC-anti-Ly-6G/Ly-6C (Gr-1) (RB6-8C5)
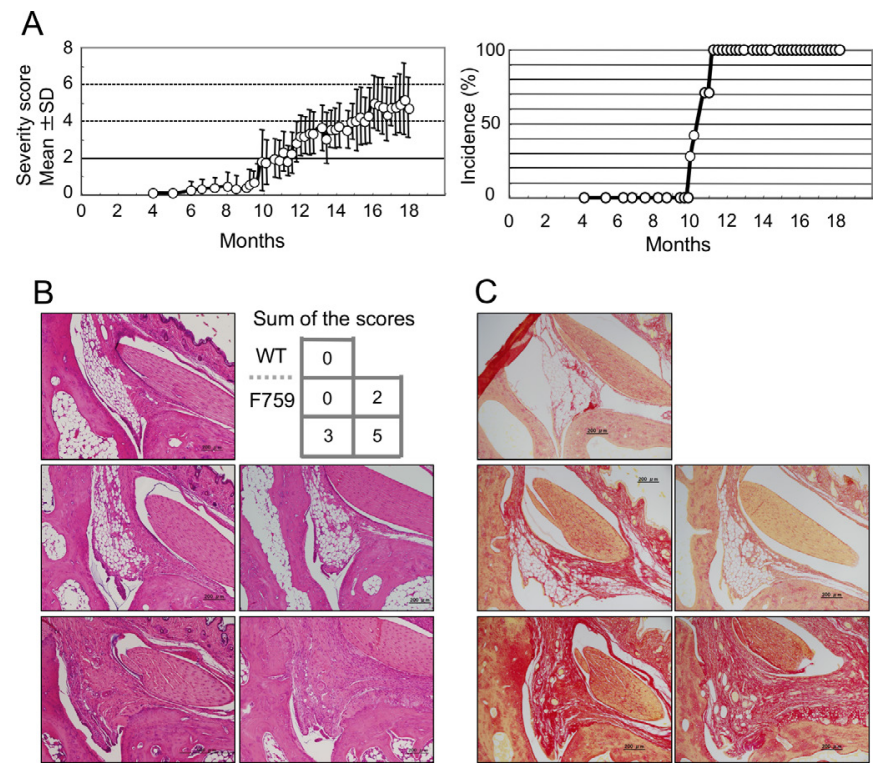

Figure 1 The clinical and histological changes of arthritis in gp130F759. (A) Incidence and severity of clinical arthritis of gp130F759 ( $n=13$; seven of male and six of female) is shown. The severity score for each mouse, the sum of scores for four limbs, was determined as described in Methods section and online supplementary figure 1. Onset of clinical arthritis was certified by the score higher than two. Severity scores are expressed as the mean $\pm S D$. (B) Representative arthritic joint sections from the mice with distinct severity scores. Slices of the joints were stained with H\&E. (C) Results of Sirius red staining of the slice specimens obtained from the same mice used in (B) are indicated. WT, wild type.
(BD biosciences), PE-anti-CD3e (145-2C11) (Tonbo Bioscience, Burlingame, California, USA), PE-Cy7-anti-TER119, APC-anti-CD11c (N418), APC-Cy7-anti-CD19 (6D5) (BioLegend), eFlour450-anti-CD4 (GK1.5) (Tonbo Bioscience) mAbs. Set 2 consists of PerCP-Cy5.5-labelled anti-CD3e, -CD19, -NK1.1 (PK136) (Tonbo Bioscience), -TER119 (eBioscience, San Diego, California, USA), PE-SiglecF (BD Biosciences), APC-F4/80 (eBioscience), APC-Cy7-Gr-1 and Pacific Blue-CD11b (BioLegend). The stained samples were analysed with FACSCanto II with FACSDiva software (BD Biosciences). Details to define cellular subsets are indicated in online supplementary figure 2. Cells were suspended in $0.4 \%$ Trypan Blue and total viable cell numbers were counted using Bürker-Türk haemocytometer under a microscope. With total viable cell numbers and frequencies obtained by flowcytometry (online supplementary figure 3 ), the cell numbers of a certain cell-lineage in the spleen or synovium were calculated.

\section{ELISA}

The protocol of anti-CCP ELISA (MESA CUP-2 test, MBL, Nagoya, Japan) was adapted for mouse serum using goat anti-mouse IgM or IgG antibodies-labelled with alkaline phosphatase (SouthernBiothech, Birmingham, Alabama, USA) as secondary antibodies. ELISA kits for mouse C reactive protein (CRP) (Helica Biosystems, Santa Ana, California, USA) and mouse RF (IgM) (FUJIFILM Wako Shibayagi, Gunma, Japan) were used according to the manufacturer's instructions.

\section{Quantitative real-time RT-PCR}

Total RNAs were extracted with TRIzol. Random hexamer-primed cDNAs were prepared using ReverTra Ace qPCR RT Kit (TOYOBO, Osaka, Japan). Specific primers for each gene were designed using Universal Primer Design Tools available on the web site of Roche Company. Real-time PCR with the SYBR Green I dye was carried out by the 7300 RealTime PCR System (Thermo Fisher Scientific). Expression levels of target cDNAs were normalised to the endogenous transcription levels of murine Actb.

\section{Western blotting}

Lysates of the joints were prepared in TNE lysis buffer $(10 \mathrm{mM}$ Tris, $150 \mathrm{mM} \mathrm{NaCl}, 1 \mathrm{mM}$ EDTA with $1 \%$ Nonidet-P40) and the $20-\mu \mathrm{g}$ equivalent whole cell crudes were boiled in sample buffer for $5 \mathrm{~min}$, resolved on Sodium dodecyl sulfate-Poly-Acrylamide Gel Electrophoresis (SDS-PAGE), and transferred electrically onto Immobilon-FL PolyVinylidene DiFluoride (PVDF) membrane (Merck Millipore, Darmstadt, Germany). After blocking with fish gelatin, the membrane was incubated with rabbit anti-PAD4 antibody (Proteintech Group, Chicago, Illinois, USA), and rabbit antiphospho-STAT3 (Tyr705) (pSTAT3) antibody (Cell Signaling Technology, Beverly, Massachusetts, USA), for overnight at $4^{\circ} \mathrm{C}$. Bound antibodies were visualised with secondary antibody IRDye 800CW-labelled 

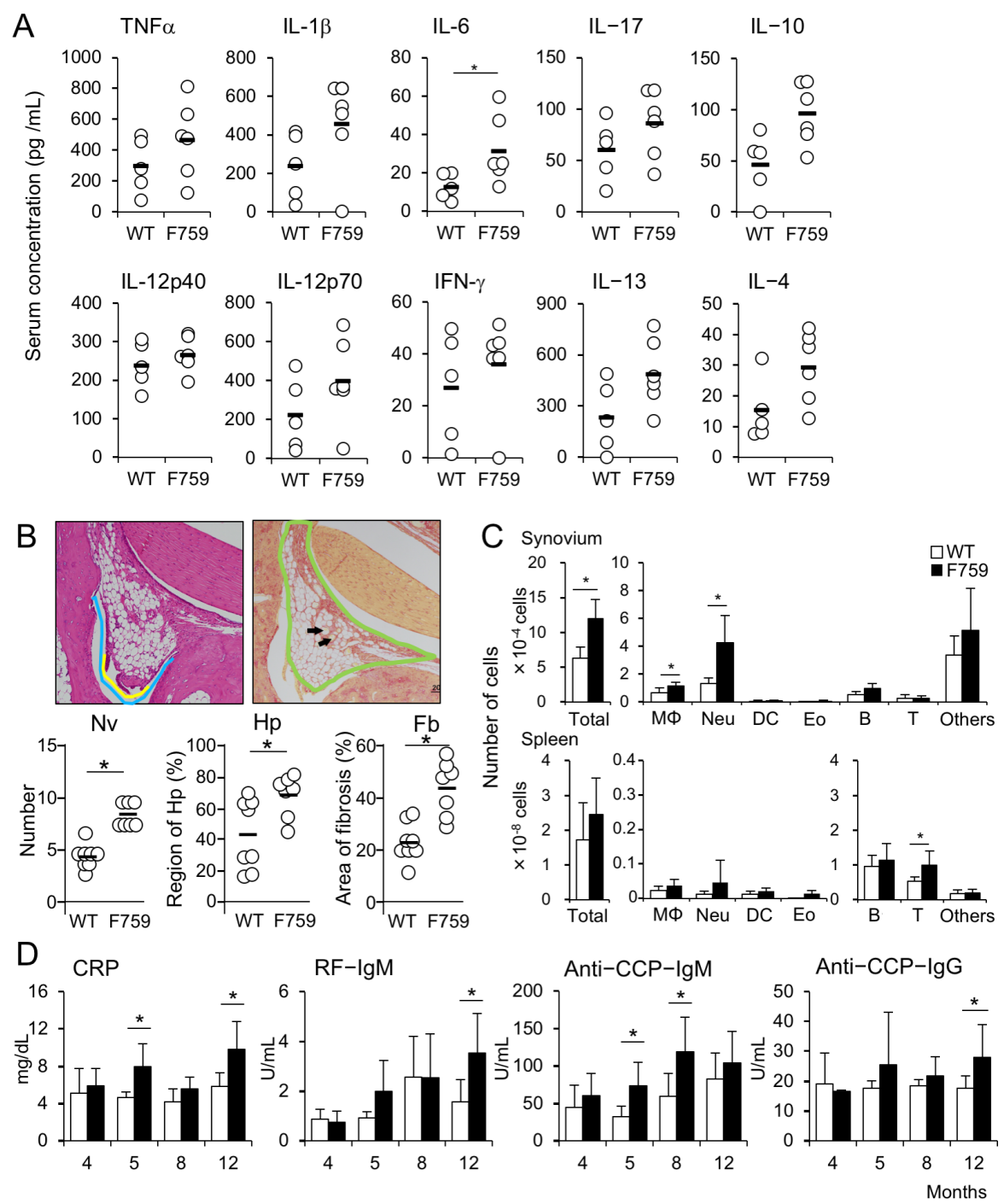

Figure 2 The earliest pathological changes of arthritis in gp130F759 develop at 5 months of age. (A) Sera were collected from WT $(n=5)$ and gp130F759 $(n=6)$, and concentrations of cytokines were measured using Bio-Plex. Black bald line shows averages and open circle shows values for individual mice. ${ }^{*} p<0.05$. (B) Histological changes in the synovium of the hindlimb joint with score 0 from wild type (WT) $(n=7)$ and gp130F759 $(n=6)$ at 5-5.5 months old were estimated by degrees of neovascularisation $(\mathrm{Nv})$, hyperplasia $(\mathrm{Hp})$ and fibrosis $(\mathrm{Fb})$ as described in Materials and methods section. The average and individual scores are indicated as horizontal bars and circles, respectively. ${ }^{*} p<0.05$. (C) Haematopoietic cell numbers in the synovium obtained by collagenase treatment and the spleen from WT (open bars) and gp130F759 (black bars) were analysed by flow cytometry. (D) Titres of CRP, RF-IgM, anti-circular citrullinated peptide (CCP)-IgM and anti-CCP-IgG antibodies in the sera of WT (open bars) and gp130F759 (black bars) were measured from 4 to 12 months old. ${ }^{*} p<0.05$. CRP, C reactive protein; $\mathrm{Fb}$, fibrosis; Hp, hyperplasia; $\mathrm{Nv}$, neovascularisation.

donkey anti-rabbit IgG (LI-COR, Lincoln, Nebraska, USA) with Odyssey CLx (LI-COR). Anti-pSTAT3 antibody was stripped with Western BLoT Stripping Buffer (TAKARA Bio, Shiga, Japan), and then the membrane was reprobed with rabbit anti-STAT3 antibody (Cell Signaling Technology).

\section{Immunohistology}

Ankle sections were made by Kawamoto's film method ${ }^{24}$ using cryofilm type 2 (Section-Lab Co, Hiroshima, Japan). The sections were fixed with cold-acetone, blocked with Phosphate buffered saline (PBS) containing 5\% donkey serum, 2\% FCS and $0.09 \% \mathrm{NaN}_{3}$, and then incubated with rabbit anti-mouse PAD4 or anti-pSTAT3 with Can
Get Signal immunostain buffer A (TOYOBO) for 2 hours at room temperature. After washed, secondary antibodies Cy3-labelled $\mathrm{F}\left(\mathrm{ab}{ }^{\prime}\right)$, donkey anti-rabbit IgG $(\mathrm{H}+\mathrm{L})$ (Jackson ImmunoResearch, West Grove, Pennsylvania, USA) with Hoechst 33342 were incubated for 30 min at room temperature. For studying colocalisation, sections were first stained with rabbit anti-PAD4 antibody and Cy3-labelled $\mathrm{F}\left(\mathrm{ab}^{\prime}\right)_{2}$ donkey anti-rabbit IgG $(\mathrm{H}+\mathrm{L})$. The sections were blocked with $5 \%$ rabbit serum, washed and then stained with biotinylated rabbit antipSTAT3 (Tyr705) monoclonal antibody (Cell Signaling Technology) and DyLight488-streptavidin (Vector Laboratories, Burlingame, California, USA). Sections were 
mounted with VECTASHIELD (Vector Laboratories), scanned with LSM700 confocal microscope (ZEISS, Jena, Germany) and analysed with Zen (ZEISS).

For preparation of cytospin slides, synovial cells were centrifuged onto slide glass with Shandon Cytospin III (Thermo Fisher Scientific) and air-dried immediately.

\section{Statistics}

Serum concentrations of cytokines, autoantibodies and CRP, cell numbers, histological and biochemical quantification, and quantity of gene expression, were compared by the Mann-Whitney U test. A part of data was compared by the Kruskal-Wallis test and Dunn's method was used as post-hoc testing. Data were analysed using GraphPad Prism8 (GraphPad Software Inc., San-Diego, CA).

\section{RESULTS}

\section{Earliest pathological changes in gp130F759 mice develop at 5} months old

We re-evaluated the clinical course of arthritis in gp130F 759 under SPF conditions using a scoring system described in Methods section and online supplementary figure 1 . As reported previously, ${ }^{17}$ obvious restriction of joint flexibility appeared at 8 months old. This gradually increased in degree, leading to ankylosis. The incidence of clinical arthritis was $50 \%$ at 11 months old and reached $100 \%$ by 12 months old (figure 1A). There were no sex differences in the age of onset, progress and incidence of arthritis.

First, we examined the relationships between clinical scores and histological changes of ankle joint specimens stained with $\mathrm{HE}$ and Sirius red (figure $1 \mathrm{~B}$ and $1 \mathrm{C}$ ). Joint specimens in a group with sum of the scores 3 showed significant changes such as thickening of the synovial lining, and replacement of adipocytes by fibroblasts, and neovascularisation. At score 5, cellular infiltration increased. In mice with scores of 0 , there were apparently no changes in gp130F759 compared with WT. These data indicate that our scoring system for clinical arthritis reflects histological changes of the joints.

To clarify the earliest changes in joints developing arthritis, we focused on the age around 5 months old, when very faint resistance in the flexibility of the ankle joints was perceived. Bio-Plex analysis of serum cytokines revealed increases in various cytokines including inflammatory (TNF $\alpha$, IL-1 $\beta$, IL-6, IL-17), anti-inflammatory (IL-10), Th1 (IL-12, IFN $\gamma$ ) and Th2 (IL-4, IL-13) cytokines (figure 2A). However, most of these changes were not significant except for IL-6 and there were no obvious histological changes at this age. Therefore, we quantitatively re-evaluated the specimens of hindlimbs from gp130F759 with clinical arthritic scores 0 using criteria indicated in figure 2B. As shown in figure 2B, increased degrees of neovascularisation, hyperplasia and fibrosis were observed in the joints of gp130F 759 with clinical arthritic scores 0, indicating that local pathological changes in the joints had already developed. This was further indicated by flow cytometry analyses of synovium, which revealed increases in total cell numbers approximately twofold including neutrophils, macrophages and others in the synovium but not in the spleen (figure 2C, online supplementary figure 3 ). These results prompted us to examine the sera to obtain data usable for diagnosis of RA. Serum CRP and anti-CCP-IgM significantly increased with non-significant increase of RF-IgM (figure 2D), indicating that gp130F759 at 5 months old could be a model equivalent to preclinical to early phase of human RA. Therefore, we analysed the synovium of gp130F759 mice around 5 months old in more detail.

\section{Padi4 gene expression was increased in the joints of gp130F759}

Since serum anti-CCP antibody increased, we examined the expression of Padi family genes by real-time PCR. Expression levels of Padi4, but not Padi1, 2, 3 and 6, were significantly higher in the joints of gp130F759 than those of WT (figure 3A, online supplementary figure 4). Furthermore, expression of $I l 6$ and Osm genes also increased in the joints (figure 3B). This strongly suggests that Padi4 gene expression is induced in the process of local inflammation mediated by neutrophils, which produce OSM. Among the chemokines known to attract neutrophils, expression of $\mathrm{Cxcl1}, \mathrm{Cxcl} 2$ and $\mathrm{Cxcl}$ did not increase but that of $\mathrm{Cxcl} 7$ significantly increased by 54 times (figure 3B).

Since expression of Padi4 gene was increased in joints of gp130F759, we examined PAD4 protein levels and activation status of STAT3 by western blot analysis. As shown in figure 4A, increases of PAD4 protein and tyrosine-phosphorylation of STAT3 in gp130F759 were clearly demonstrated, suggesting the possible relationship between the local increase of Padi4 expression and abnormally enhanced activation of STAT3 in the joints of gp130F759. To confirm the spatial corelation of STAT3 activation and PAD4 production in the joint microenvironments, immunohistochemical analyses were performed using frozen sections of the joints of gp130F 759 at 5 months old. Increased reactivities of anti-PAD4 antibody as well as those of anti-phospho-STAT3 antibody were clearly detectable in gp130F759 (figure 4B). Furthermore, the reactivities of anti-phospho-STAT3 antibody, some of which showed nuclear localisation (inset), were increased in gp130F759. These data clearly demonstrate that aberrant STAT3 activation and PAD4 production take place in the preclinical stage of arthritis in gp130F759. Importantly, very few cells, with frequency approximately two in 340 cells, showed colocalisation of reactivities of anti-phospho-STAT3 antibody and anti-PAD4 antibody (figure 4B, right inset), indicating that a relationship between gp130 signal and PAD4 production is observable in a single cell level.

Then, we investigated cells expressing Padi4 in the joints. In the synovia of gp130F759, Padi4 gene was highly expressed selectively by haematopoietic cells (figure 4C). Interestingly, increased expression of $I l 6$ gene was observed in non-haematopoietic synovial cells in gp130F759. Actually, ELISA revealed increased production of IL-6 in culture supernatant fluid (CSF) of 

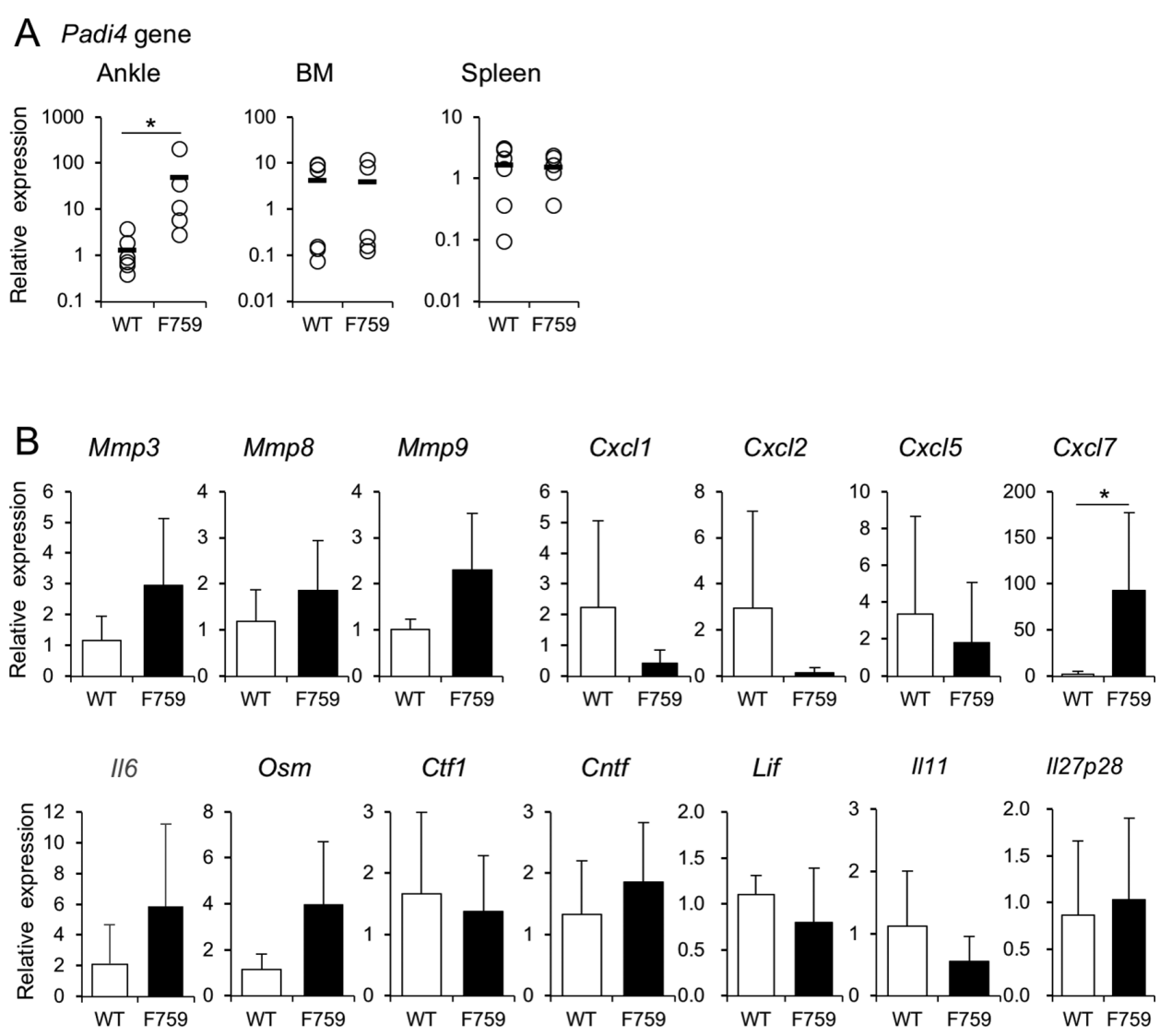

Figure 3 Padi4 gene expression was increased in the ankle of gp130F759. (A) Expression levels of Padi4 gene were quantified by real-time PCR in the ankle joints, bone marrow or spleen of wild type (WT) and gp130F759 at 5 months old ( $n=6$ each) are shown. The black bar shows average and the open circle shows individual value. (B) Gene expression levels of Mmps, IL-6 family cytokines and chemokines attracting neutrophils, in the joints of wild type $(n=6)$ and gp130F759 $(n=6)$ at 5 months old were quantified. The average and SD are indicated as bar graph. Open bars and black bars indicate WT and gp130F759, respectively. ${ }^{*} \mathrm{p}<0.05$.

CD45 ${ }^{-}$synovial cells from gp130F759 compared with that of WT (figure 4D). Next, to characterise the haematopoietic cells expressing PAD4, synovial cells liberated with collagenase treatment were cytospun and stained with anti-PAD4 antibody. Characteristic nuclear morphology of PAD $4^{+}$cells indicated that they are neutrophil lineage (figure $4 \mathrm{E}$ photo and inset). Numbers of $\mathrm{PAD} 4^{+}$cells were higher in gp130F759 than WT mice (figure 4E graph).

To examine whether the increased expression of Padi4 depends on IL-6, neutrophils enriched from the bone marrow were stimulated with IL-6 (online supplementary figure 5). The expression levels of Padi4 in neutrophils from gp130F759 and WT similarly increased by IL-6 stimulation.

To confirm the interaction between non-haematopoietic synovial cells and neutrophils, we tested whether the CSF from non-haematopoietic synovial cells has activity to induce Padi4 expression in neutrophils. Stimulation with CSF of CD45 synovial cells from gp130F759 induced Padi4 gene expression in WT neutrophils (figure 4F). This Padi4-inducing activity was proved to be mediated mainly by IL-6, because preincubation with anti-IL-6 neutralising antibody but not control antibody inhibited Padi4 gene induction to the basal level. To confirm operation of IL-6 signal in a single cell level, WT neutrophils were incubated with CSF of $\mathrm{CD}^{-} 5^{-}$synovial cells from gp130F759 for $30 \mathrm{~min}$, cytospun, and stained with rabbit anti-phosph-STAT3 antibody with Cy3 labelled second antibody. Photos in figure 4G clearly indicate that the CSF strongly induced phosphorylation of STAT3, which is inhibited by pretreatment of CSF with anti-IL-6 neutralising antibody but not control IgG1. Thus, from these data, we conclude that Padi4 expression was induced in neutrophils by IL-6 produced from non-haematopoietic synovial cells in the joint microenvironment of gp130F759.

\section{Operation of the IL-6-PAD4 axis in preclinical arthritis}

To clarify the causative, in vivo roles for IL-6 in the increases of PAD4 and anti-CCP antibody, IL-6 was deleted by crossing gp130F759 with Il6KO. Complete deletion of $I l 6$ gene in gp130F759 resulted in normalisation of splenomegaly (figure 5A,C), and serum levels of RF and anti-CCP antibodies (figure 5B). Importantly, $\mathrm{PAD} 4$ production (figure $5 \mathrm{D}$ ) and synovial cell numbers (figure 5E) returned to almost normal levels. These data indicate that Padi4 induction in the synovium is IL-6 dependent and that operation of the IL-6-PAD4 axis in 
A

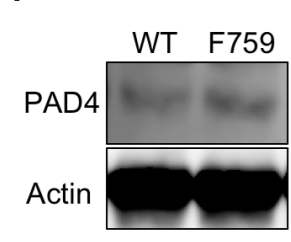

pSTAT3

STAT3

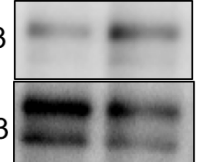

B
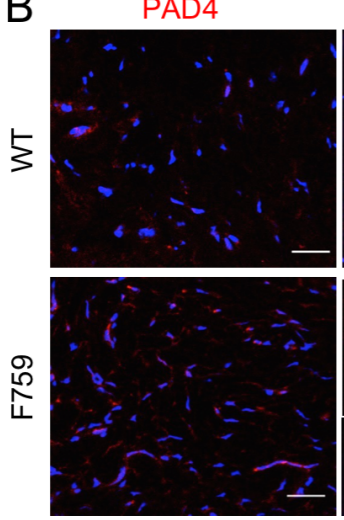

pSTAT3

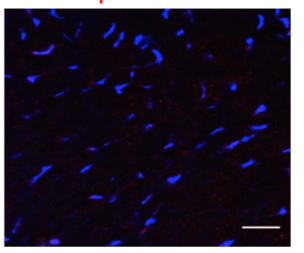

PAD4

pSTAT3

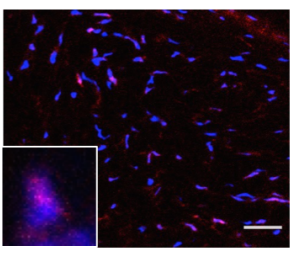

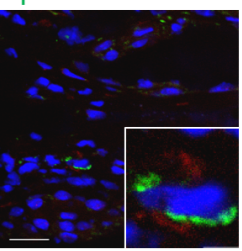

C
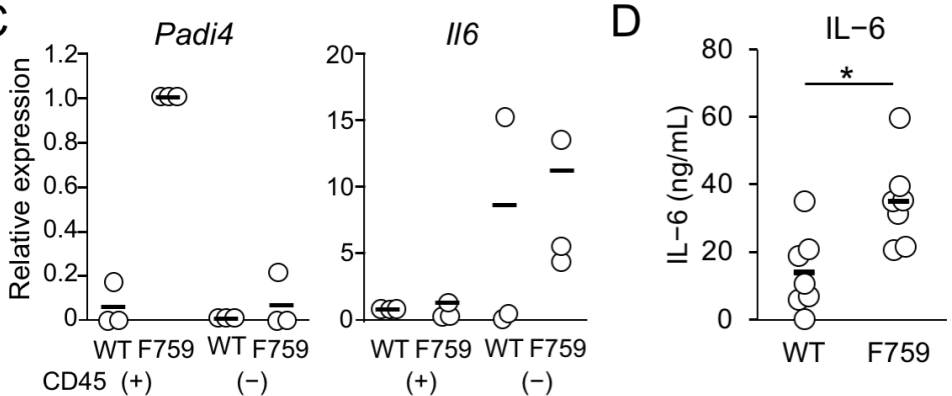

$(+)$

$(-)$
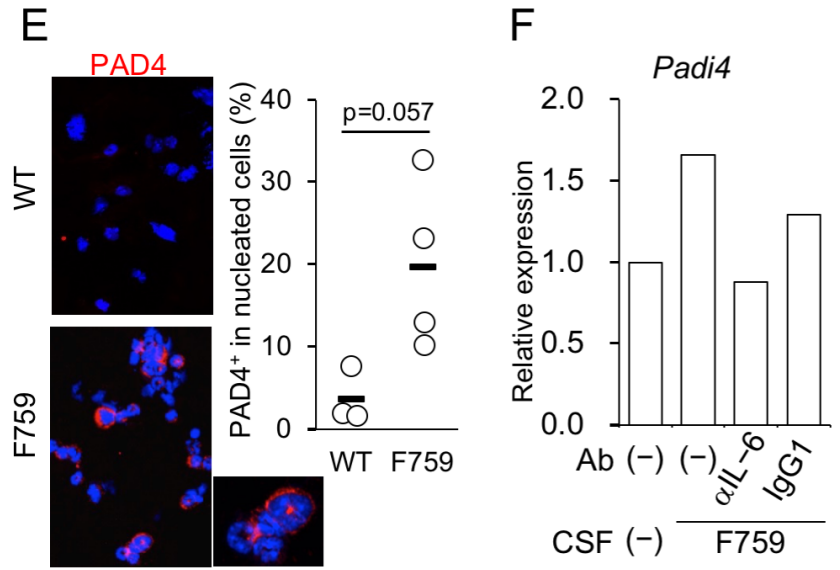

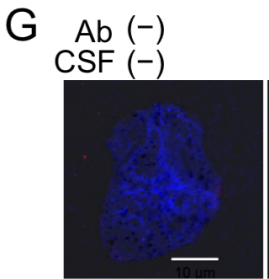

Ab IgG1

CSF F759

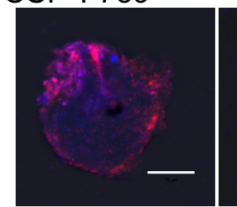

$(-)$

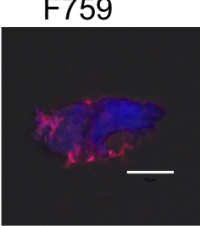

$\alpha \mathrm{lL}-6$

F759

Figure 4 PAD4 $^{+}$cells in the synovium of gp130F759 are neutrophils. (A) Amounts of PAD4 protein and STAT3 phosphorylation in the wrist joints from wild type (WT) and gp130F759. Lysates of the joints from three mice of each genotype were pooled and subjected to western blotting. (B) Frozen sections of the synovium from WT and gp130F759 were stained with anti-PAD4 and pSTAT3 antibodies and Cy3-labelled F $\left(a b^{\prime}\right)_{2}$ donkey anti-rabbit lgG $(\mathrm{H}+\mathrm{L})$. Representative pictures from four independent experiments are shown. Nuclei were stained with Hoechst 33342. Colocalisation was examined with anti-PAD4 and biotinylated pSTAT3 antibodies, which were visualised with Cy3-labelled anti-Rabbit IgG and Alexa488-streptavidin, respectively. A PAD4 producing cell whose STAT3 is phosphorylated (inset). The bars indicate $20 \mu \mathrm{m}$. (C) Gene expression levels of Padi4 and I/6 in haematopoietic $\left(C D 45^{+}\right)$or non-haematopoietic $\left(C D 45^{-}\right)$synovial cells which were separated with rat anti-mouse CD45 antibody and sheep anti-rat IgG magnetic beads. Summarised data from three independent experiments are shown. (D) IL-6 concentration in supernatant of primary culture of CD45 synovial cells from WT and gp130F759 at 5 months old ( $n=7$ ). ${ }^{*} \mathrm{p}<0.05$. (E) Morphology of synovial cells producing PAD4. The photos of representative cytospin specimens from WT and gp130F759 are shown. Synovial cells cytospun onto the slide glass were incubated with anti-PAD4 antibody (red) and Hoechst 33342 for nuclear staining (blue). In the graph, black bar shows average and the open circle shows individual value for each mouse. (F) IL-6 produced by CD45 ${ }^{-}$synovial cells induced Padi4 expression in neutrophils. WT bone marrow neutrophils were stimulated for 6 hours with culture supernatant fluid (CSF) from gp130F759 pretreated with anti-IL-6 antibody or control IgG1. Then RNA from the neutrophils were prepared and transcription of Padi4 was estimated by real-time PCR using specific primers and SYBR green. Relative expression levels compared with Actb are shown. (G) IL-6 in the CSF from gp130F759 induced activation of STAT3 in neutrophils. WT bone marrow neutrophils were stimulated for 30 min with CSF from gp130F759 pretreated with anti-IL-6 antibody or control IgG1. The neutrophils were cyto-spun, air-dried and stained with anti-pSTAT3 antibody and Cy3-labelled donkey anti-rabbit IgG antibody. Pictures were taken with LSM700 confocal microscope. The bars indicate $10 \mu \mathrm{m}$. 

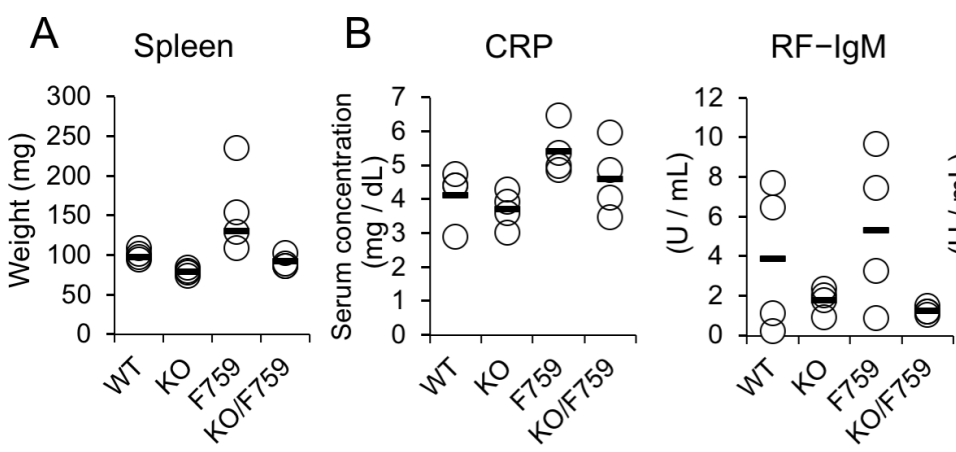

Anti-CCP-IgM
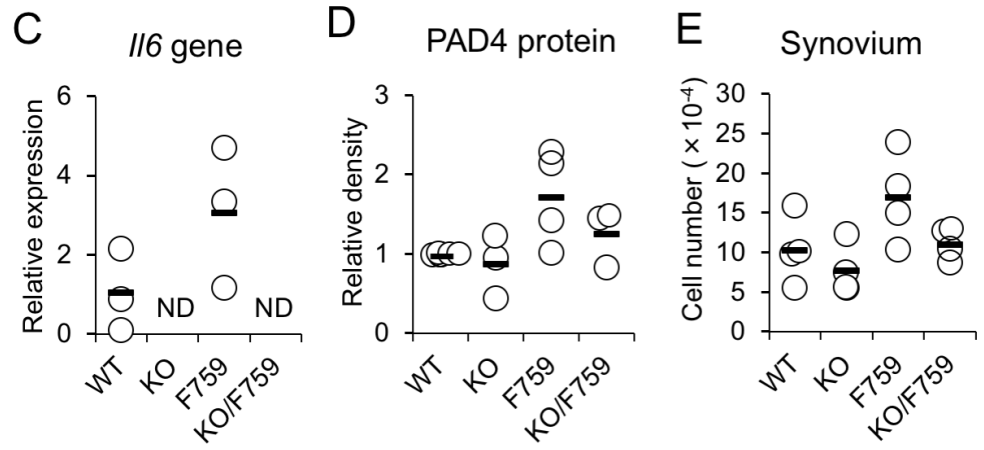

Figure 5 IL-6-dependent changes in preclinical phase of arthritis in gp130F759. Multiple parameters of WT, I/6KO (abbreviated as KO), gp130F759 (F759) and II6KO/gp130F759 (KO/F759) at 5-6 months old ( $n=4$ each) were examined. As systemic changes, (A) weight of the spleen, (B) serum titres of CRP, RF-IgM, and anti-CCP IgM are indicated at top. As a local changes in the joints, (C) I/6 gene expression by real time PCR, (D) the relative amounts of PAD4 protein by western blot \{the average ratio of band densities (PAD4/ $\beta$-actin) of $W T=1.0\}$ and $(E)$ synovial cell numbers are indicated at bottom. Statistical significance was estimated by the Kruskal-Wallis test and Dunn's method. ND; not detectable. CCP, circular citrullinated peptide; CRP, $C$ reactive protein.

vivo at an early preclinical phase of RA-like arthritis in gp130F759 (figure 6).

\section{DISCUSSION}

In this study, we focused on the natural course of RA-like arthritis in gp130F759, and especially on events during the preclinical phase. To understand kinetics of systemic immune or inflammatory responses, we first examined a set of serum cytokines with Bio-Plex. This revealed increases of multiple cytokines at 5 months old, much before the recognition of definitive, clinical arthritis at around 8 months old. In most of the cytokines, levels of increases were less than twofold, and not statistically significant. This is possibly because the initial age and progress of preclinical changes in each mouse are variable in the spontaneous arthritis in gp130F759, which is contrasted with other arthritis models induced by immunisation with type II collagen or antigen. Since this time point, 5 months old, coincides with appearance of very faint resistance of joint flexibility much less than point 1 of the arthritis score, we extended the study around this age.

Increases of CRP, anti-CCP antibody and RF in the sera were detected at 5 months old, suggesting that as far as the laboratory data, gp130F759 around 5 months old is equivalent to fulfil the criteria of RA. Flow cytometrical analyses revealed increases of the haematopoietic cells competent in innate immunity. Although the clinical signs of arthritis were not obvious at 5 months old, quantitative histopathological analyses provided evidence for synovial hyperplasia and neovascularisation, the latter is also characteristic of early RA. ${ }^{25}$ Thus, it can be said that gp130F759 at 5 months old is a model for the preclinical to early phase of RA when minute pathological changes begin. Gene expression analyses revealed that at this time point, increases of $M m p 3$ and Il6 started in the joint, indicating that local inflammatory responses had already begun. Importantly, the expression of Padi4 gene among members of Padi gene family was increased selectively in the joint. Increased production of PAD4 protein with increase of STAT3 phosphorylation was confirmed by biochemical as well as immunohistological measurements. The technique for magnetic cell separation revealed the role-sharing by non-haematopoietic cells expressing $I l 6$ and haematopoietic cells expressing Padi4 in the synovial microenvironment. Cytological analyses revealed that PAD4 producing cells are neutrophils and stimulation of IL-6 induced Padi4 gene expression in neutrophils. All of these data suggest that the IL-6-PAD4 axis participates in the preclinical phase of RA-like arthritis in gp130F759. We have verified the operation of the IL-6-PAD4 axis by four facts; increased production of IL-6 in CSF of CD45 synovial cells from gp130F759, induction of STAT3 phosphorylation and Padi4 transcription in WT neutrophils stimulated with the CSF, and both inductions were inhibited by anti-IL-6 neutralising antibody.

In the promoter region of Padi4, presence of conserved binding sites for NF-אB, TATA-ETS-like, AP-1, Krüppel-like factor-6 (KLF-6) and SP1 are reported. ${ }^{26}$ Lack of typical STAT3 binding elements suggests that STAT3 indirectly 


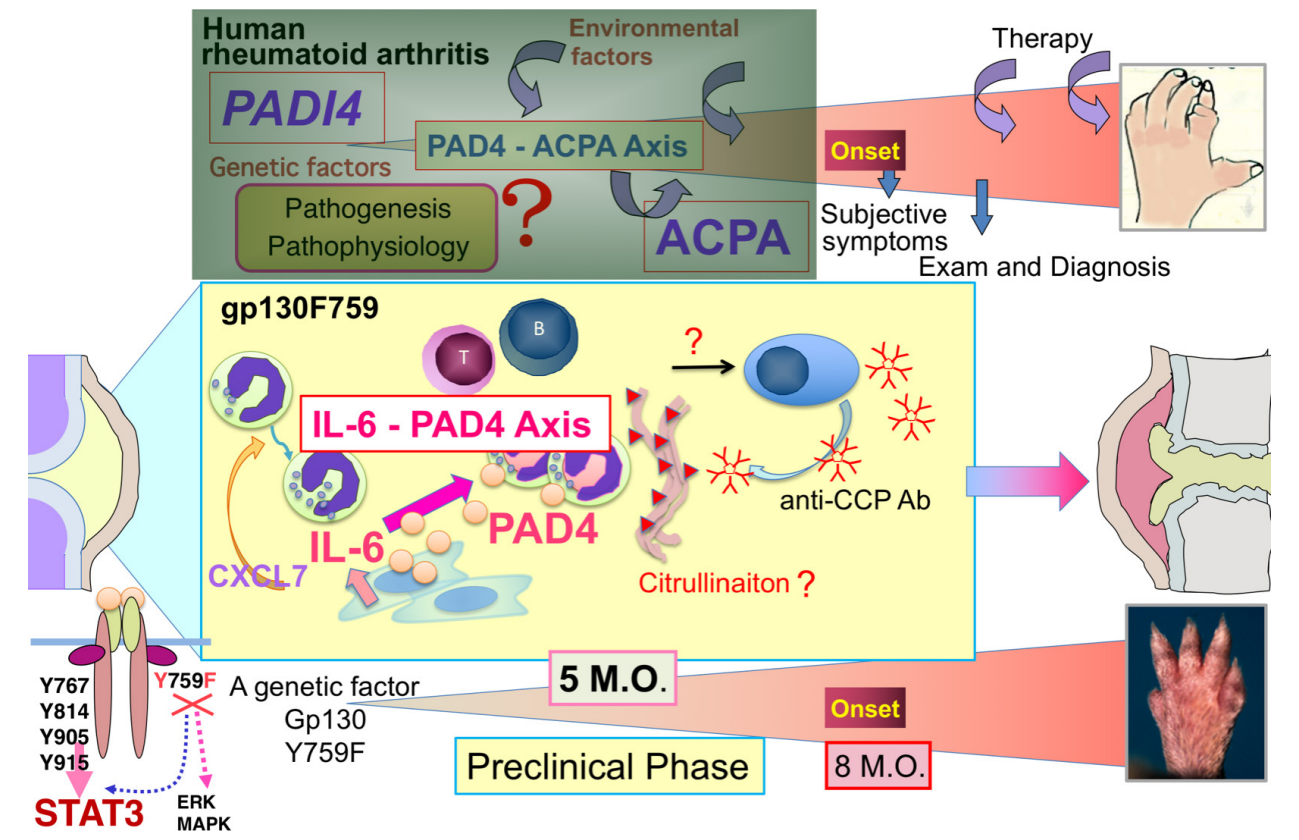

Figure 6 The IL-6-PAD4 axis in the synovium of preclinical phase of rheumatoid arthritis (RA)-like arthritis in gp130F759. anti-citrullinated peptide autoantibodies (ACPAs) are detectable much earlier than onset of RA. Presence of ACPA indicates that tolerant self-antigens have been modified by citrullination and that the modified self-antigens become recognised as foreign by adaptive immunity. Thus, ACPA and anti-circular citrullinated peptide (CCP) antibody, which is adapted for clinical examination to detect ACPA as a whole, are useful biomarkers to predict development of RA. In other words, anti-CCP antibody can be used to define the preclinical phase of RA by the criteria: anti-CCP antibody positive and lack of clinical symptoms of arthritis. Since the natural course of RA is now appreciated as (1) preclinical RA, (2) early 'clinically evident' RA and (3) chronic 'established' RA, ${ }^{7}$ the molecular events and causal relationships between them in preclinical RA are gathering great attention. But the information of synovial microenvironment in preclinical RA is hardly obtainable from human. Using the mutant gp130 knock-in mice, gp130F759, we report here that gp130F759 around 5 months old could be an ideal model for the pathological phases spanning preclinical to early clinically evident RA. During this time window, the IL-6-PAD4 axis is generated by interaction of non-haematopoietic cells, most likely fibroblast-like synoviocytes, and neutrophils, representative of innate immunity, attracted by CXCL7. ERK, Extracellular Signal-regulated Kinase; MAPK, Mitogen-activated Protein Kinase; M.O.; months old.

activates transcription of Padi4. The transcriptional activator KLF-6 that is induced by gp130/STAT3 signal may play a role in inducing Padi4 transcription as reported in oligodendrocytes. ${ }^{27}$ Since there is a NF- $\kappa \mathrm{B}$ binding site and serum TNF $\alpha$ was slightly increased in gp130F759, there is possibility that Padi4 may be induced by TNF $\alpha$. But it is not likely in the case of gp130F759 around 5 months old, because increases of Tnfgene expression were not observed in $\mathrm{CD} 45^{-}$as well as CD $45^{+}$synovial cells, and TNF $\alpha$ was not detectable in culture supernatant fluid of CD45 synovial cells by ELISA (data not shown). These results have clearly indicated the causative role for IL-6 from CD $45^{-}$synovial cells to induce Padi4 transcription in neutrophils, although it cannot completely rule out possible involvement of other cytokines in vivo.

Although the promoter region of PADI4 is highly conserved among species, TNF $\alpha / \mathrm{NF}-\kappa \mathrm{B}$ signal in myeloid cells shows positive and negative regulations on Padi4 transcription in mouse and human, respectively. ${ }^{26}$ It can be said that our data have provided experimental evidences that IL-6 signal can activate Padi4 transcription, which was unpredictable by bioinformatic analyses. Previously, roles for synovial fibroblasts-neutrophil interactions in promoting adaptive immunity in RA was reported. ${ }^{28}$ Thus, the IL-6-PAD4 axis could be a critical, prerequisite event by synovial fibroblasts-neutrophil interactions to promote transition from innate to adaptive immunity in early RA. It is intriguing to speculate that the molecular mechanism triggering initiation of RA is $\mathrm{PADI} 4$ induction in neutrophils by IL-6/STAT3 signalling provided from non-haematopoietic cells, such as fibroblast-like synoviocytes, in the synovial microenvironments (figure 6). This hypothetical mechanism deserves further analyses in human system.

Deletion of IL-6 in gp130F759 was previously found to result only in retardation of the progress and reduction of severity of arthritis. However, the final incidence of disease was unaffected. ${ }^{18}$ In the preclinical phase of gp130F759 in this study, deletion of IL-6 resulted in the reduction of splenomegaly, CRP, RF, PAD4 production, anti-CCP-IgM and synovial cell numbers, indicating that these parameters are IL-6-dependent and that IL-6 is critically involved in the initiation of synovial hyperplasia.

In addition to novel findings concerning the IL-6-PAD4 axis, serum cytokine profiling also revealed other important characteristics of the preclinical phase that has similarity with RA. First, in the preclinical phase, when 
the elevation of anti-CCP antibody was detected, systemic, low-level increases of typical inflammatory cytokines, such as TNF $\alpha$, IL-1 $\beta$, IL-6 and IL-17 already existed. Second, cytokines that are elevated in this phase showed no deviation to Th1 or Th2, that is, both Th1 and Th2 types were elevated. Third, an immune-suppressive cytokine, IL-10 also increased. None of the changes were specific to this animal model. On the contrary, the pre-clinical phase of gp130F759 has quite similar cytokine profile to the early phase of RA. ${ }^{29-32}$ Especially, TNF $\alpha$, IL-1 $\beta$, IL-10 and IL-13 are cytokines frequently reported in early RA and also detected in our model. As a STAT3-activating cytokine, IL-10 may have a unique role in early $\mathrm{RA}^{25}$ and dysregulation of IL-10 in RA synovial macrophages has been reported. ${ }^{33}$

Another similarity to RA is induction of a chemokine CXCL7 attracting neutrophils, which was reported to be produced by synovial macrophages in early RA. ${ }^{34}$ Interestingly, our novel, infection-induced arthritis model using gp130F759 at 3 months old (unpublished observation by Yahagi et al.) revealed that systemic infection of Mycoplasma fermentans induced expression of $\mathrm{Cxcl} 7$ and accumulation of cells dominated by neutrophil in the synovium. This suggests intriguing possibility that aberrant cytokine signals and triggering by microorganisms utilise common pathway to initiates arthritis by induction of $\mathrm{Cxcl} 7$ and accumulation of neutrophils in gp130F759.

From all the data obtained in this paper, we propose a model for molecular events in the synovial microenvironments of pre-clinical phase of RA. Importantly, the IL-6-PAD4 axis operates to promote the transition from innate immunity to acquired immunity (figure 6). Thus, gp130F759 is useful to learn the molecular mechanisms for heterogenous pathways leading to a clinical entity RA, which shows variable clinical courses and responses to therapies.

\section{Author affiliations \\ ${ }^{1}$ Immunology and Molecular Genetics, Kawasaki Medical School, Kurashiki, Japan ${ }^{2}$ Otorhinolaryngology, Kawasaki Medical School, Kurashiki, Japan \\ ${ }^{3}$ Rheumatology, Kawasaki Medical School, Kurashiki, Japan \\ ${ }^{4}$ R\&D Division, Ayumi Pharmaceutical Corp, Kyoto, Japan \\ ${ }^{5}$ Nara Research and Development Center, Santen Pharmaceutical Co Ltd, Nara, Japan \\ ${ }^{6}$ Department of Clinical Research, National Hospital Organization, Osaka Minami Medical Center, Kawachinagano, Japan}

Acknowledgements We thank Dr M. Kopf for generous gift of II6KO. We thank Dr P. W. Kincade for critical reviewing the manuscript. We specially thank Mr N. Iwachido, Ms E. Ohtsuki and Ms K. Yamane at Kawasaki Medical School Central Research Institute for excellent technical assistances of histological analyses and confocal microscopy, respectively. We also thank Ms R. Tanaka, Y. Kenmotsu, M. Funatsu and Y. Matsuo for their technical assistance in this study.

Contributors AY, YS and $\mathrm{KI}$ contributed to the conception and study design. AY, TS, MT-I, HI and KI acquired and analysed the data. AY, TS, HH, MT-I, HI, YS and KI contributed to interpretation of the data. AY and KI wrote the first version of the manuscript and $\mathrm{FT}, \mathrm{HA}, \mathrm{MI}, \mathrm{YM}, \mathrm{HI}$ and $\mathrm{YS}$ revised it critically. All authors read and approved the final manuscript.

Funding This work was supported by JSPS KAKENHI Grant Numbers JP17K16217 to AY and JP21590448, JP17K08798 to KI. This work was also supported by Research Project Grants from Kawasaki Medical School to AY and KI and The KAWASAKI Foundation for Medical Science \& Medical Welfare to AY.
Competing interests $\mathrm{HH}$ and YM have received research support from Chugai pharmaceutical Co.

Patient consent for publication Not required.

Ethics approval The study was approved by the Animal Care and Use Committee of Kawasaki Medical School.

Provenance and peer review Not commissioned; externally peer reviewed.

Data sharing statement All data relevant to the study are included in the article or uploaded as supplementary information.

Open access This is an open access article distributed in accordance with the Creative Commons Attribution Non Commercial (CC BY-NC 4.0) license, which permits others to distribute, remix, adapt, build upon this work non-commercially, and license their derivative works on different terms, provided the original work is properly cited, appropriate credit is given, any changes made indicated, and the use is non-commercial. See: http://creativecommons.org/licenses/by-nc/4.0/.

\section{REFERENCES}

1. van Gaalen FA, Linn-Rasker SP, van Venrooij WJ, et al. Autoantibodies to cyclic citrullinated peptides predict progression to rheumatoid arthritis in patients with undifferentiated arthritis: a prospective cohort study. Arthritis Rheum 2004;50:709-15.

2. van Venrooij WJ, van Beers JJBC, Pruijn GJM. Anti-CCP antibody, a marker for the early detection of rheumatoid arthritis. Ann N Y Acad Sci 2008;1143:268-85.

3. van Venrooij WJ, van Beers JJBC, Pruijn GJM. Anti-CCP antibodies: the past, the present and the future. Nat Rev Rheumatol 2011;7:391-8.

4. Aletaha D, Neogi T, Silman AJ, et al. 2010 rheumatoid arthritis classification criteria: an American College of Rheumatology/ European League against rheumatism collaborative initiative. Arthritis Rheum 2010;62:2569-81.

5. Mclnnes IB, Schett G. Pathogenetic insights from the treatment of rheumatoid arthritis. Lancet 2017;389:2328-37.

6. Mclnnes IB, Schett $\mathrm{G}$. The pathogenesis of rheumatoid arthritis. $N$ Engl J Med 2011;365:2205-19.

7. Firestein GS, Mclnnes IB. Immunopathogenesis of rheumatoid arthritis. Immunity 2017;46:183-96.

8. Smolen JS, Aletaha D, Barton A, et al. Rheumatoid arthritis. Nat Rev Dis Primers 2018;4.

9. Vossenaar ER, Zendman AJW, van Venrooij WJ, et al. Pad, a growing family of citrullinating enzymes: genes, features and involvement in disease. Bioessays 2003;25:1106-18.

10. Suzuki A, Yamada R, Chang X, et al. Functional haplotypes of PADI4 encoding citrullinating enzyme peptidylarginine deiminase 4 , are associated with rheumatoid arthritis. Nat Genet 2003;34:395-402.

11. Suzuki A, Kochi Y, Shoda $H$, et al. Decreased severity of experimental autoimmune arthritis in peptidylarginine deiminase type 4 knockout mice. BMC Musculoskelet Disord 2016;17.

12. Seri $Y$, Shoda $H$, Suzuki $A$, et al. Peptidylarginine deiminase type 4 deficiency reduced arthritis severity in a glucose-6-phosphate isomerase-induced arthritis model. Sci Rep 2015;5.

13. Shelef MA, Sokolove J, Lahey LJ, et al. Peptidylarginine deiminase 4 contributes to tumor necrosis factor $\alpha$-induced inflammatory arthritis. Arthritis Rheumatol 2014;66:1482-91.

14. Ishihara K, Hirano T. IL-6 in autoimmune disease and chronic inflammatory proliferative disease. Cytokine Growth Factor Rev 2002;13:357-68.

15. Ishihara K, Hirano T. Molecular basis of the cell specificity of cytokine action. Biochim Biophys Acta 2002;1592:281-96.

16. Ohtani $T$, Ishihara $K$, Atsumi $T$, et al. Dissection of signaling cascades through gp130 in vivo: reciprocal roles for STAT3- and Shp2mediated signals in immune responses. Immunity 2000;12:95-105.

17. Atsumi T, Ishihara K, Kamimura D, et al. A point mutation of Tyr-759 in interleukin 6 family cytokine receptor subunit gp130 causes autoimmune arthritis. J Exp Med 2002;196:979-90.

18. Sawa S, Kamimura D, Jin G-H, et al. Autoimmune arthritis associated with mutated interleukin (IL)-6 receptor gp130 is driven by STAT3/IL-7-dependent homeostatic proliferation of CD4+ T cells. J Exp Med 2006;203:1459-70.

19. Ishihara K, Sawa S, Ikushima $\mathrm{H}$, et al. The point mutation of tyrosine 759 of the IL-6 family cytokine receptor gp130 synergizes with HTLV$1 \mathrm{pX}$ in promoting rheumatoid arthritis-like arthritis. Int Immunol 2004;16:455-65.

20. Murakami M, Okuyama $\mathrm{Y}$, Ogura $\mathrm{H}$, et al. Local microbleeding facilitates IL-6- and IL-17-dependent arthritis in the absence of tissue antigen recognition by activated T cells. J Exp Med 2011;208:103-14. 
21. Ogura $\mathrm{H}$, Murakami M, Okuyama $\mathrm{Y}$, et al. Interleukin-17 promotes autoimmunity by triggering a positive-feedback loop via interleukin-6 induction. Immunity 2008;29:628-36.

22. Murakami M, Hirano T. A four-step model for the IL-6 amplifier, a regulator of chronic inflammations in tissue-specific MHC class IIassociated autoimmune diseases. Front Immunol 2011;2.

23. Kopf M, Baumann H, Freer G, et al. Impaired immune and acute-phase responses in interleukin-6-deficient mice. Nature 1994;368:339-42.

24. Kawamoto T. Use of a new adhesive film for the preparation of multipurpose fresh-frozen sections from hard tissues, whole-animals, insects and plants. Arch Histol Cytol 2003;66:123-43.

25. Tsubaki T, Arita N, Kawakami T, et al. Characterization of histopathology and gene-expression profiles of synovitis in early rheumatoid arthritis using targeted biopsy specimens. Arthritis Res Ther 2005; 7:R825-36.

26. Abbas AK, Le K, Pimmett VL, et al. Negative regulation of the peptidylarginine deiminase type IV promoter by NF-KB in human myeloid cells. Gene 2014;533:123-31.

27. Laitman BM, Asp L, Mariani JN, et al. The transcriptional activator Krüppel-like factor-6 is required for CNS myelination. PLoS Biol 2016;14:e1002467.

28. Carmona-Rivera C, Carlucci PM, Moore E, et al. Synovial fibroblastneutrophil interactions promote pathogenic adaptive immunity in rheumatoid arthritis. Sci Immunol 2017;2. doi:10.1126/sciimmunol. aag3358

29. Hueber W, Tomooka BH, Zhao X, et al. Proteomic analysis of secreted proteins in early rheumatoid arthritis: anti-citrulline autoreactivity is associated with up regulation of proinflammatory cytokines. Ann Rheum Dis 2007;66:712-9.

30. Khan IH, Krishnan VV, Ziman M, et al. A comparison of multiplex suspension array large-panel kits for profiling cytokines and chemokines in rheumatoid arthritis patients. Cytometry B Clin Cytom 2009;76:159-68.

31. Deane KD, O'Donnell Cl, Hueber W, et al. The number of elevated cytokines and chemokines in preclinical seropositive rheumatoid arthritis predicts time to diagnosis in an age-dependent manner. Arthritis Rheum 2010;62:3161-72.

32. Gómez-Puerta JA, Celis R, Hernández MV, et al. Differences in synovial fluid cytokine levels but not in synovial tissue cell infiltrate between anti-citrullinated peptide/protein antibody-positive and -negative rheumatoid arthritis patients. Arthritis Res Ther 2013;15.

33. Antoniv TT, Ivashkiv LB. Dysregulation of interleukin-10-dependent gene expression in rheumatoid arthritis synovial macrophages. Arthritis Rheum 2006:54:2711-21.

34. Yeo L, Adlard N, Biehl M, et al. Expression of chemokines CXCL4 and CXCL7 by synovial macrophages defines an early stage of rheumatoid arthritis. Ann Rheum Dis 2016;75:763-71. 